\title{
Erratum
}

\section{Erratum for: Analysis of softwares for emotion recognition in children and teenagers with autism spectrum disorder}

In the article, "Analysis of softwares for emotion recognition in children and teenagers with autism spectrum disorder" with DOI number: 10.1590/1982-0216/201921112318, published in the journal Revista Cefac, 21(1):e12318, in author name:

Where it was:

Paula Dornhoffer Paro Costa

Read:

Paula Dornhofer Paro Costa 